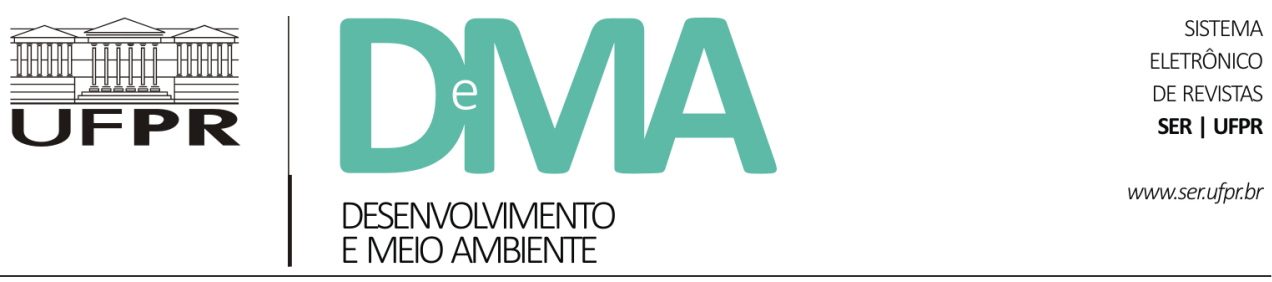

\title{
Onde estão os dados para o Planejamento Espacial Marinho (PEM)? Análise de repositórios de dados marinhos e das lacunas de dados geoespaciais para a geração de descritores para o PEM no Sul do Brasil
}

\section{Where are the Data Needed for Marine Spatial Planning (MSP) in Brazil? Analysis of Marine Data Repositories and Geospatial Data Gaps for the Generation of Descriptors for MSP in Southern Brazil}

\author{
Tiago Borges Ribeiro GANDRA ${ }^{1,2^{*}}$, Jarbas BONETTI ${ }^{2}$, Marinez Eymael Garcia SCHERER ${ }^{2}$ \\ ${ }^{1}$ Instituto Federal de Educação, Ciência e Tecnologia do Rio Grande do Sul (IFRS), Rio Grande, RS, Brasil. \\ ${ }^{2}$ Universidade Federal de Santa Catarina (UFSC), Florianópolis, SC, Brasil. \\ *E-mail de contato: tiago.gandra@riogrande.ifrs.edu.br
}

Artigo recebido em 31 de agosto de 2017, versão final aceita em 20 de novembro de 2017.

\begin{tabular}{ll}
\hline RESUMO: & O Planejamento Espacial Marinho (PEM) é um processo público de análise e alocação das atividades humanas \\
no mar e se iniciou no Brasil em 2011, mas obteve avanços incipientes. A avaliação da disponibilidade de \\
dados ambientais e de atividades humanas no mar e a identificação das lacunas em sua distribuição espacial \\
são etapas iniciais do PEM. Este trabalho utiliza uma lista de descritores para o PEM, analisa a disponibilidade \\
destes em repositórios de dados nacionais e internacionais e mostra lacunas de dados na Zona Econômica- \\
Exclusiva (ZEE) do Sul do Brasil. Os descritores necessários para o PEM foram relacionados aos temas de \\
dados geoespaciais disponíveis na Infraestrutura para Informações Espaciais na Europa (INSPIRE) para a \\
quantificação da relevância de cada tema. Os temas disponíveis na INSPIRE contribuem para a elaboração de \\
85\% dos descritores para o PEM na Europa, e os mais representativos são Zonas de Restrição, Regulação e \\
Manejo, Regiões Marinhas, Infraestrutura Industrial e de Produção, Áreas Protegidas e Habitats e Biótopos. \\
A Infraestrutura Nacional de Dados Espaciais (INDE) contribui com apenas cinco dos 145 descritores. O \\
Banco Nacional de Dados Oceanográficos (BNDO) possui uma estrutura limitada, que não o configura como \\
sendo uma plataforma para consulta de dados, sendo apenas um repositório. Mapas de densidade kernel foram \\
utilizados para avaliar a distribuição e detectar lacunas de dados geoespaciais. Os dados disponíveis possuem, \\
em geral, maior concentração na plataforma continental interna (até 50 m) e apresentam descontinuidades \\
na distribuição espacial variadas para cada tema. É importante avançar no PEM brasileiro, mesmo com os
\end{tabular}


problemas de escassez, dispersão ou ausência de dados. São necessários adaptações e esforços institucionais para que a INDE agregue dados marinhos no Brasil. Estratégias claras são necessárias para que as instituições passem a alimentar com maior efetividade os repositórios nacionais e internacionais, qualificando o processo e garantindo a possibilidade de incorporar a melhor informação científica disponível ao PEM.

Palavras-chave: Infraestrutura de Dados Espaciais (IDE); Gestão Costeira Integrada (GCI); conservação de habitats marinhos; exploração econômica dos oceanos; análise espacial; INSPIRE.

ABSTRACT: Marine Spatial Planning (MSP) is a public process of analyzing and allocating the spatial and temporal distribution of human activities in marine areas. In Brazil it has started in 2011, but with little improvements. One of the first steps for MSP is the assessment of data on environmental and human activities, as well as data gaps identification. This article uses a list of descriptors required for MSP, analyzes the availability of data in national and international data repositories, and shows geospatial data gaps in the southern Brazilian Economic-Exclusive Zone (EEZ). The 145 descriptors listed were related to themes available in the Infrastructure for Spatial Information in Europe (INSPIRE) to assess the relevance of each theme for MSP. The INSPIRE themes contribute to $85 \%$ of the MSP descriptors and the most relevant themes are management, restriction and regulation zones, sea regions, production and industrial facilities, protected sites and habitats and biotopes. The Brazilian National Spatial Data Infrastructure (INDE, in Portuguese) contributes with only five of the 145 descriptors. The National Oceanographic Data Bank (BNDO, in Portuguese) has a structure that inhibits its use as a platform for data query, being only a repository. The Kernel Density Estimator (KDE) was used to assess geospatial data gaps in the studied area. The available data have, in general, greater concentration on the internal continental shelf (up to $50 \mathrm{~m}$ ), with distinctive gaps in each theme. It is important to advance in the Brazilian MSP, even with the problems of scarcity, data dispersion or lack of data. Adaptations and efforts are needed to make possible the marine data aggregation at INDE. Government needs to advance in MSP and encourage institutions to increase the marine data feeding in national and international repositories, qualifying the process and ensuring the possibility of incorporating the best scientific information available to MSP.

Keywords: Spatial Data Infrastructure (SDI); Integrated Coastal Management (ICM); marine habitat conservation; ocean resources exploitation; spatial analysis; INSPIRE.

\section{Introdução}

O Planejamento Espacial Marinho (PEM) é um processo público de análise e alocação das atividades humanas no oceano (Ehler \& Douvere, 2009) que teve início no Brasil em 2011, restrito, todavia, a iniciativas locais (de Freitas et al., 2014). O PEM é uma estratégia explícita e tangível em direção à redução dos conflitos entre diferentes grupos de usuários (Collie et al., 2013) e à conservação da biodiversidade. A considerável extensão e a alta complexidade de ecossistemas, usos e conflitos na zona marinha do Brasil fazem com que seja urgente o início do PEM no Brasil.

Os habitats e processos marinhos são mais dinâmicos e tridimensionais que os terrestres, sendo mais difícil delimitar e mapear os ecossistemas. Dados marinhos são geralmente mais escassos, de difícil obtenção e, muitas vezes, de representatividade espacial e temporal mais limitada do que os dados terrestres. Aspectos físicos, como tipo de sedimento, batimetria, concentração de clorofila e massas 
d'água, podem ser utilizados como indicadores (proxies) para a definição de sistemas ambientais marinhos, uma vez que modulam a configuração dos usos antrópicos e naturais. As atividades humanas e infraestrutura afetam o ambiente marinho, mas também possuem sua presença ou ausência determinada pelo tipo de sistema ecológico e condições ambientais (Douvere et al., 2007).

Devido aos altos custos de obtenção e tratamento de dados e às características do ambiente marinho, é importante definir quais são os tipos de dados prioritários para o PEM, bem como identificar as lacunas de dados geoespaciais e de séries históricas. Shucksmith et al. (2014) relatam que a coleção de dados e mapeamento é uma das etapas que mais consume tempo no PEM, mas, depois da criação de uma plataforma para administração dos mesmos, a coleção de dados subsequentes foi mais eficiente. Portanto, é de fundamental importância a definição de diretrizes de padronização e sistematização de dados nas etapas iniciais do PEM.

A seleção, a aquisição e a organização dos dados marinhos, bem como a definição das técnicas de harmonização dos dados disponíveis, são grandes limitações para a elaboração do PEM (Halpern et al., 2012; European Commission, 2016).

Alguns dos componentes críticos para o PEM são:

(1) triagem e coleção de dados geoespaciais, (2) gerenciamento de dados, (3) análise de dados e (4) sistemas de suporte à decisão (Stamoulis \& Delevaux, 2015). Apesar de serem etapas sequenciais, a definição do sistema de suporte à decisão a ser uti- lizado tem influência na seleção dos dados a serem coletados e gerenciados. Portanto, bases de dados bem consolidadas, integradas e multidisciplinares são um primeiro passo em direção ao PEM, mas a sua construção deve levar em conta as metodologias de análise e suporte à decisão disponíveis ou desejáveis.

Uma Infraestrutura de Dados Espaciais (IDE) abrange a tecnologia, as políticas, os padrões, os recursos humanos e as atividades relacionadas necessários para adquirir, processar, distribuir, usar, manter e preservar dados geoespaciais (Masser, 2005) e, portanto, vai além de ser um repositório ou plataforma on-line de dados.

Existem vários exemplos de repositórios e IDEs para os oceanos, dos quais podemos citar o British Oceanographic Data Center (BODC) ${ }^{1}$, o Centro Argentino de Datos Oceanográficos ${ }^{2}$, o Australian Ocean Data Network (AODN) ${ }^{3}$, o norte-americano National Centers for Environmental Information (NCEI) ${ }^{4}$ a Infrastructure for Spatial Information in Europe (INSPIRE) ${ }^{5}$. Estas IDEs diferem tanto nos tipos de dados disponíveis quanto na forma de acesso e de distribuição destes dados. Neste artigo, a INSPIRE foi adotada como exemplo para comparação com a situação brasileira, pois várias iniciativas para o PEM na Europa utilizaram a INSPIRE como base. Além disto, a Comissão Europeia elaborou uma lista dos descritores necessários para o PEM e a relacionou com as camadas de informação (CIs) disponíveis na INSPIRE (European Commission, 2016).

\footnotetext{
${ }^{1}$ http://www.bodc.ac.uk/

${ }^{2}$ http://www.hidro.gov.ar

${ }^{3} \mathrm{http}: / /$ portal.aodn.org.au

${ }^{4} \mathrm{http}: / /$ www.nodc.noaa.gov

${ }^{5} \mathrm{http}$ ://inspire.ec.europa.eu
} 
No Brasil, a Infraestrutura Nacional de Dados Espaciais (INDE) foi lançada em 2010 visando à integração entre sistemas de diferentes instituições. Tem o propósito de catalogar, integrar e harmonizar dados geoespaciais existentes nas instituições do governo brasileiro. Possui boa documentação e padrões (standards) definidos para os dados e metadados.

O governo brasileiro iniciou o processo de elaboração do PEM em 2011, entretanto, até o momento se obtiveram avanços apenas pontuais, para pequenas áreas do extenso litoral brasileiro (de Freitas et al., 2014). No sul do país, foram encontradas duas iniciativas para o PEM, porém, em escala local em regiões costeiras. Gern et al. (2017) utilizaram o Zoneamento Portuário e Aquaviário de Itajaí como base do PEM por meio da aplicação de alguns princípios do PEM. O Projeto Babitonga Ativa $^{6}$ vem mapeando e promovendo a gestão participativa dos serviços ecossistêmicos da Baía Babitonga por meio de oficinas e reuniões com os atores sociais que se beneficiam diretamente da região, avançando no PEM local.

Este trabalho utiliza a lista de descritores para o PEM (European Commission, 2016) e analisa a disponibilidade destes descritores para o Brasil, indicando potencialidades e restrições dos repositórios de dados analisados. Além disto, identifica dados prioritários para o PEM e lacunas de dados geoespaciais disponíveis para a Zona Econômica-Exclusiva (ZEE) do sul do Brasil.

\section{Metodologia}

Os dados de entrada associados à construção de cada tema de informação geoespacial foram obtidos por meio dos metadados e das especificações da INSPIRE. As listas de descritores e temas (e seus atributos) foram inseridas no sistema gerenciador de banco de dados (SGBD) PostgreSQL e os relacionamentos (do tipo muitos para muitos - M:N) entre estas duas tabelas implementados por meio de SQL. Os temas mais relevantes foram definidos com o cálculo do número de relacionamentos com os descritores para o PEM (Figura 1).

O levantamento de dados geoespaciais na INDE foi realizado por meio da consulta espacial utilizando retângulos envolventes (coordenadas máximas e mínimas) exclusivamente nas áreas marinhas. As CIs que possuíam apenas feições terrestres foram desconsideradas na análise. É importante notar que este tipo de consulta deixa de fora CIs que não possuem dados espaciais disponíveis na INDE para download ou visualização em mapas interativos.

Posteriormente, foram realizadas buscas de dados geoespaciais marinhos em outros repositórios nacionais e internacionais (Tabela 1) e definidos aqueles que poderiam ser utilizados para a obtenção de descritores necessários para o PEM. Quando disponíveis para download, estes dados foram obtidos e sistematizados em um banco de dados PostgresSQL 9.3. O processamento e a seleção espacial para manter apenas os registros de ocorrência na ZEE do sul do Brasil foram realizados por meio de consultas ao banco de dados.

${ }^{6} \mathrm{http}: / /$ www.babitongaativa.com 


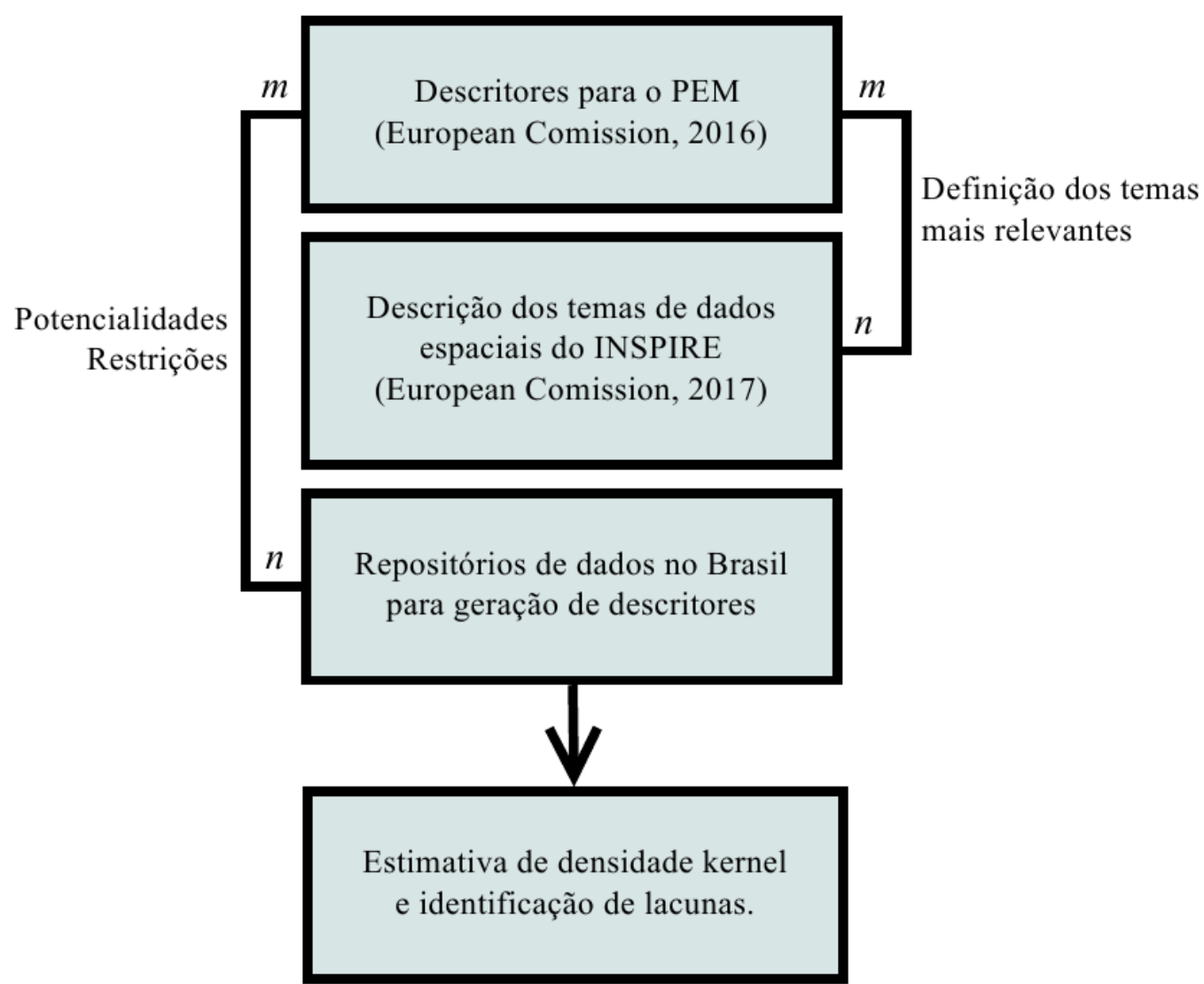

FIGURA 1 - Metodologia para priorização de descritores para o PEM no Brasil e identificação de lacunas espaciais.

FONTE: Elaboração própria.

A fim de avaliar a efetiva distribuição espacial dos dados disponíveis, visando à identificação de lacunas e de setores cuja densidade de dados é insatisfatória, foram elaborados mapas para cada conjunto de dados sistematizados com o uso do método de Estimação de Densidade Kernel, com largura de banda de $0,2^{\circ}(\sim 22 \mathrm{~km})$ e resolução espacial de $0,02^{\circ}(\sim 2 \mathrm{~km})$, utilizando os pacotes "KernSmooth" e "Raster" no software R. Para a composição e o layout final dos mapas foi utilizado o software QGIS 2.18.
Métodos de análise exploratória destes dados foram testados (análise de agrupamento, análise de componentes principais) de forma a transformar dados em informação e geração de descritores para o PEM. A análise de tais resultados foge do escopo do presente artigo, mas os aspectos relativos à sua obtenção e alguns desses resultados podem ser verificados em Gandra et al. (2017).

A análise exploratória dos dados obtidos e a observação de aspectos importantes para repositórios de dados (forma de pesquisa e distribuição de 
dados, formato, definição de padrões e metadados) foram utilizadas para a elaboração de uma lista de potencialidades e restrições dos repositórios analisados.

\section{Resultados e discussão}

\subsection{Temas de dados espaciais da INSPIRE mais utilizados para o PEM}

A INSPIRE une legislação, diretivas e especificações técnicas, dados e serviços de dados espaciais para a União Europeia (European Commission, 2017). Desde 2007 foram feitos grandes esforços de integração, triagem e harmonização de dados espaciais na INSPIRE, possibilitando a utilização de dados transfronteiriços numa mesma base espacial e conceitual.

A lista da CE (European Commission, 2016) define 145 descritores para o PEM, divididos em cinco classes: limites administrativos $(\mathrm{n}=5$ descritores), informação física, química e biológica (42), usos e atividades (65), política espacial (5) e dados socioeconômicos (5). Aproximadamente $85 \%$ dos descritores $(n=123)$ podem ser obtidos por meio de 21 temas disponíveis na INSPIRE. Os temas mais utilizados para o PEM são: Zonas de Restrição, Regulação e Manejo (em 20 descritores), Regiões Marinhas (16), Infraestrutura Industrial e de Produção (16), Áreas Protegidas (12) e Habitats e Biótopos (11) (Figura 2).

Os temas relacionados a um maior número de descritores devem ser priorizados para o avanço do PEM no Brasil.

\subsection{Temas e descritores disponiveis para o PEM no Brasil}

$\mathrm{Na}$ INDE, foram encontradas somente cinco camadas de informação com registros em áreas marinhas: (a) Unidades de Conservação ( $\mathrm{MMA}^{7}$ ), relacionada ao tema "Áreas Protegidas", (b) Cartas Náuticas Digitais $\left(\mathrm{DHN}^{8}\right)$, elacionada com o tema "Elevação", (c) Portos, relacionada às "Redes de Transporte", (d) Áreas Prioritárias para a Conservação da Biodiversidade (MMA, 2007) e (e) Bentos da Zona Costeira e Marinha do Brasil, ambas relacionadas indiretamente com os temas "Distribuição de Espécies" e "Habitats e Biótopos". Devido a esta carência de dados na INDE, outras bases de dados nacionais (Tabela 1) e globais (Tabela 2) foram consultadas e analisadas quanto às potencialidades e restrições para a geração de descritores para o PEM no Brasil.

\subsubsection{Relevo submarino}

Mapas batimétricos são a base para a definição de diversos descritores para o PEM, além de serem utilizados para elaboração de outros temas, como "Habitats e biótopos" e "Regiões marinhas". No Brasil, a melhor fonte para dados batimétricos na plataforma continental são as Cartas Náuticas, disponibilizadas pela Diretoria de Hidrografia e Navegação (DHN) da Marinha.

Uma limitação das Cartas Náuticas é a densidade desigual dos pontos disponíveis. Estas possuem articulação irregular e priorizam o mapeamento em fundeadouros e áreas portuárias,

${ }^{7}$ Ministério do Meio Ambiente.

${ }^{8}$ Diretoria de Hidrografia e Navegação - Marinha do Brasil. 
pois têm como objetivo a segurança à navegação. vocação náutica, existem importantes lacunas de Assim, sobretudo em setores próximos à costa sem dados batimétricos no país.

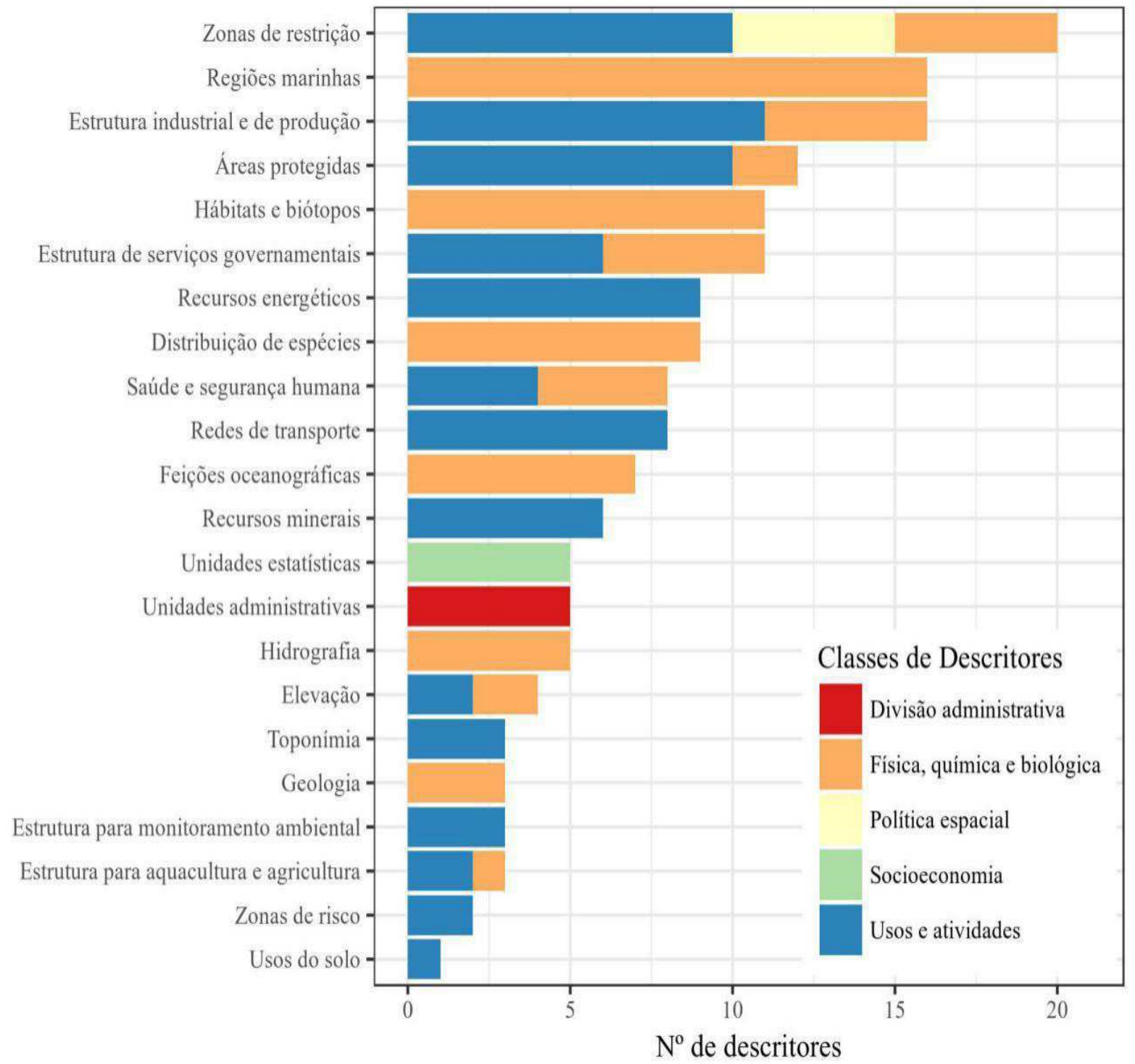

FIGURA 2 - Número de descritores para o PEM formados por cada camada de informação do INSPIRE. FONTE: Elaboração própria 
TABELA 1 - Bases de dados nacionais, limitações e potencialidades para utilização no PEM

\begin{tabular}{|c|c|c|c|}
\hline Bases de dados & Descritores & & Restrições (R) e Potencialidades (P) \\
\hline $\begin{array}{c}\text { Unidades de } \\
\text { Conservação (I3GEO) }\end{array}$ & Áreas Protegidas & $\mathrm{P}$ & $\begin{array}{l}\text { Arquivos vetoriais com os limites de todas as unidades de } \\
\text { conservação nos três níveis de governo. }\end{array}$ \\
\hline $\begin{array}{l}\text { Cartas Náuticas } \\
\text { Digitais }^{10}\end{array}$ & $\begin{array}{l}\text { Relevo submarino; batimetria; cala- } \\
\text { do para portos; plataformas; boias; } \\
\text { áreas restritas para navegação }\end{array}$ & $\mathrm{P}$ & $\begin{array}{l}\text { Boa informação de batimetria para áreas portuárias. Cober- } \\
\text { tura de boa parte da ZEE brasileira. Diferentes escalas. }\end{array}$ \\
\hline $\begin{array}{l}\text { Programa de Monitora- } \\
\text { mento de Embarcações } \\
\text { Pesqueiras (PREPS) })^{11}\end{array}$ & $\begin{array}{l}\text { Número de embarcações de pesca } \\
\text { industrial; áreas importantes para a } \\
\text { pesca (por modalidade, estação) }\end{array}$ & $\mathrm{P}$ & $\begin{array}{l}\text { Dados de rastreamento possibilitam a criação de diversos } \\
\text { descritores. Censo da pesca industrial. Dados vetoriais das } \\
\text { zonas de exclusão de pesca. }\end{array}$ \\
\hline \multirow{2}{*}{$\begin{array}{l}\text { Banco de Dados de } \\
\text { Exploração e Produção } \\
\qquad(B D E P)^{12}\end{array}$} & \multirow{2}{*}{$\begin{array}{l}\text { Áreas de extração/concessão de pe- } \\
\text { tróleo e gás; Plataformas de explo- } \\
\text { ração. }\end{array}$} & $\mathrm{P}$ & Dados oficiais de blocos e rodadas de concessão \\
\hline & & $\mathrm{R}$ & $\begin{array}{l}\text { Falta de alimentação após criação, dados desatualizados. } \\
\text { Dados de potencial exploração não estão disponíveis }\end{array}$ \\
\hline \multirow{2}{*}{$\begin{array}{l}\text { Banco Nacional de } \\
\text { Dados Oceanográfico } \\
(\text { BNDO })^{13}\end{array}$} & \multirow{2}{*}{$\begin{array}{c}\text { Tipos de fundo; relevo submarino, } \\
\text { estações meteorológicas e maregrá- } \\
\text { ficas. }\end{array}$} & $\mathrm{P}$ & $\begin{array}{l}\text { Repositório oficial brasileiro. Coleciona e padroniza diver- } \\
\text { sos tipos de dados oceanográficos. Reúne dados de diversas } \\
\text { instituições. }\end{array}$ \\
\hline & & $\mathrm{R}$ & $\begin{array}{l}\text { Distribuição de dados por meio de solicitação por e-mail. } \\
\text { Dificuldade de pesquisa de dados disponíveis. Grandes la- } \\
\text { cunas espaciais. Metadados incompletos. }\end{array}$ \\
\hline $\begin{array}{l}\text { Sistema de Apoio ao } \\
\text { Monitoramento de } \\
\text { Mamíferos Aquáticos } \\
\text { (SIMMAM) })^{14}\end{array}$ & Rotas de migração (mamíferos) & $\mathrm{P}$ & $\begin{array}{l}\text { Repositório oficial do ICMBio e da rede de encalhes de ma- } \\
\text { míferos. Dados disponíveis para download. Reúne dados de } \\
\text { diversas instituições. Dados de encalhes, avistagens e cap- } \\
\text { tura incidental. Metadados. }\end{array}$ \\
\hline
\end{tabular}

FONTE: Elaboração própria.

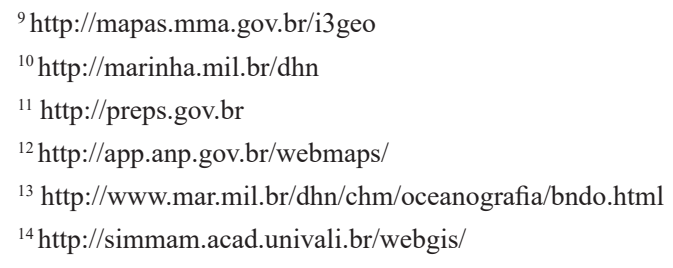


TABELA 2 - Bases de dados globais, limitações e potencialidades para utilização no PEM

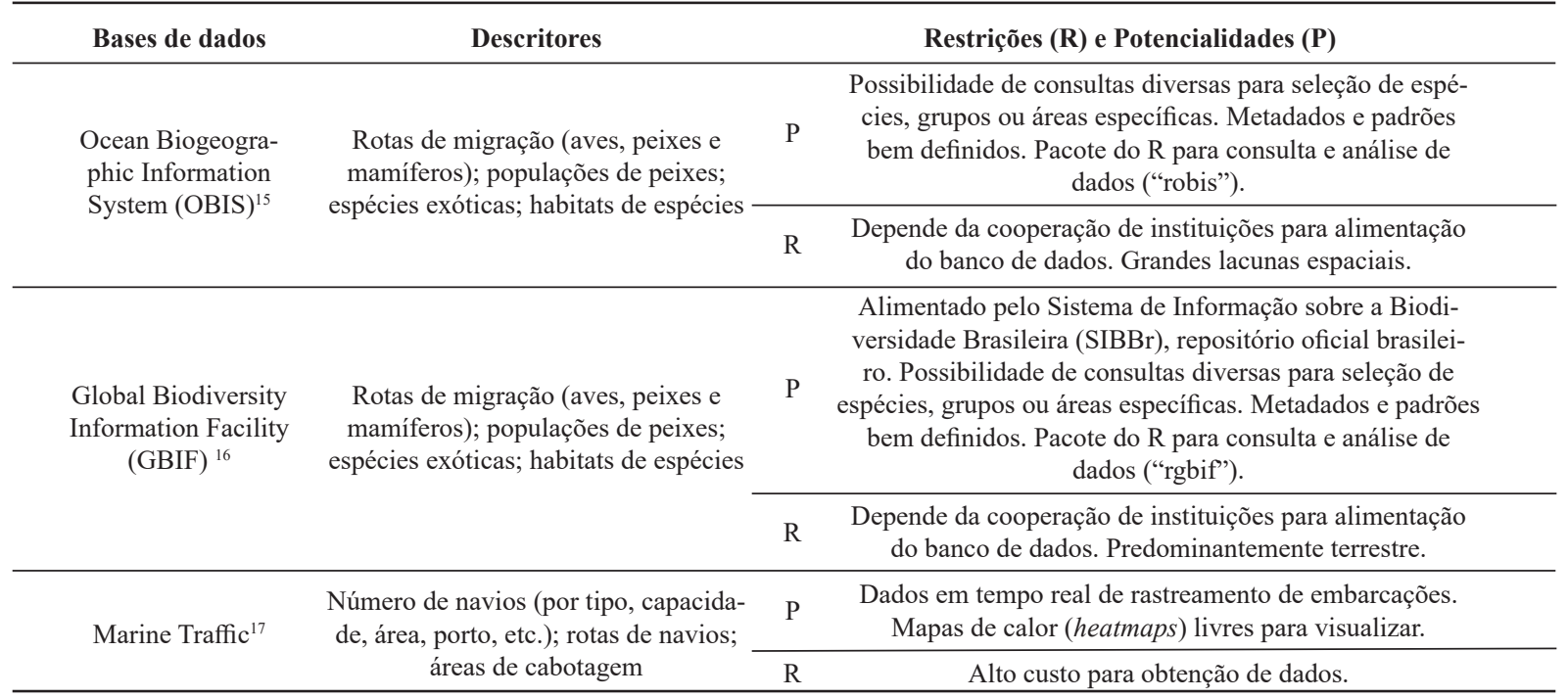

FONTE: Elaboração própria.

Além disto, as Cartas Náuticas são disponibilizadas na forma de figuras georreferenciadas e não como um modelo digital de elevação (MDE), que permitiria a utilização em ferramentas de suporte à decisão. Portanto, não existem dados oficiais com uma superfície contínua para batimetria. A construção do MDE pode ser feita por meio da vetorização manual dos pontos batimétricos das Cartas Náuticas e posterior interpolação, um processo que consome muito tempo e acumula erros e incertezas, diminuindo a qualidade da informação. Cabe mencionar que os pontos batimétricos das Cartas Náuticas foram digitalizados e integram a base de dados do Sistema de Modelagem Costeira (SMC-Brasi1 ${ }^{18}$ ), estando dis- poníveis para utilização por meio desta ferramenta.

Dados de profundidade também estão disponíveis no Banco Nacional de Dados Oceanográficos (BNDO). A análise das lacunas espaciais destes dados na ZEE do sul do Brasil mostra baixa densidade no talude e na bacia oceânica e boa distribuição na plataforma continental, com setores de alta densidade na área costeira ao norte de Florianópolis (SC) até São Francisco do Sul (SC) (Figura 3-G). Entretanto, a acurácia dos valores de profundidade não foi avaliada neste trabalho, aspecto relevante considerando-se a conhecida incompatibilidade entre os data verticais de diferentes cartas náuticas e destas com as cartas topográficas do IBGE.

\footnotetext{
${ }^{15} \mathrm{http}: / /$ www.iobis.org/

${ }^{16}$ https://www.gbif.org/

${ }^{17}$ http://www.marinetraffic.com

${ }^{18} \mathrm{http}: / /$ smcbrasil.ihcantabria.com/
} 


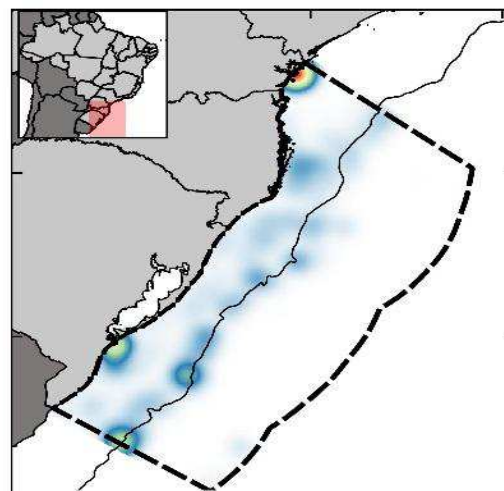

(A) OBIS: Bentônicos ( $\mathrm{N}=8030)$

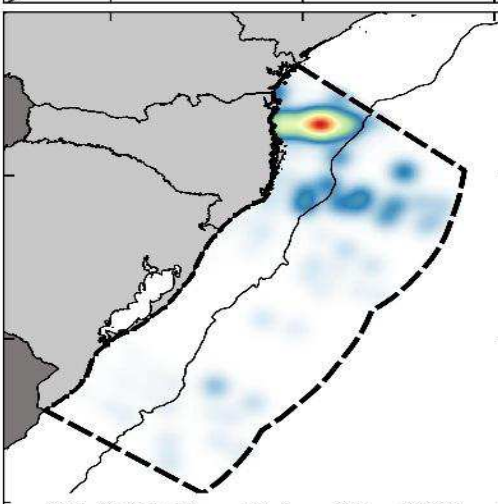

(B) OBIS: Planctônicos ( $\mathrm{N}=6858)$

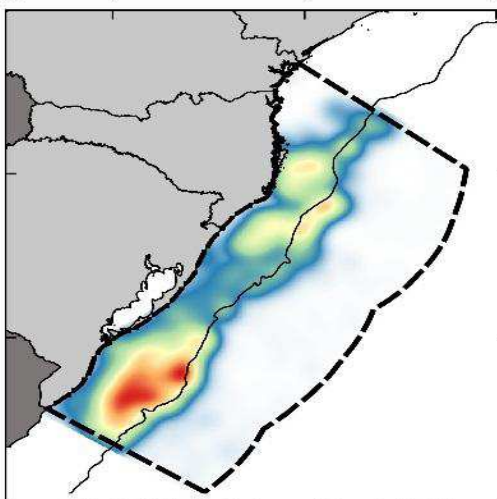

(C) OBIS: Pelágicos $(\mathrm{N}=31010)$

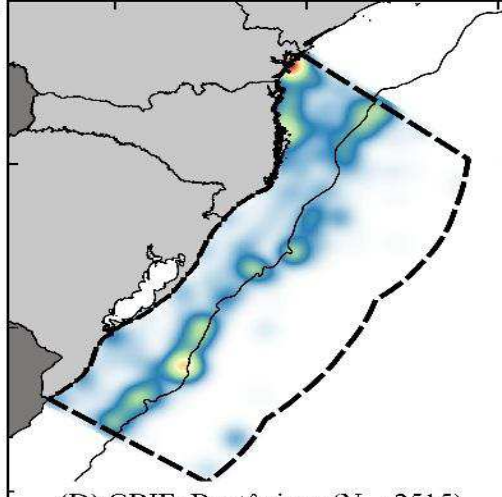

(D) GBIF: Bentônicos ( $\mathrm{N}=2515)$

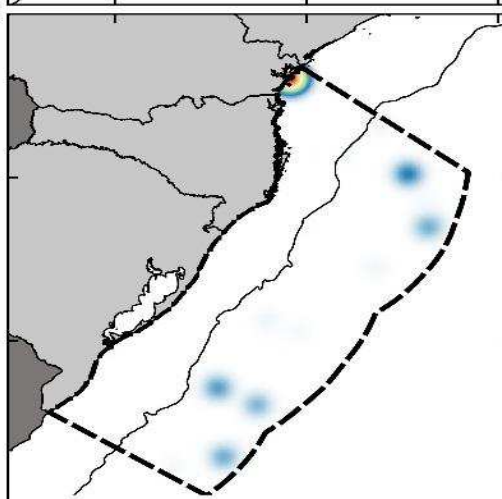

(E) GBIF: Planctônicos ( $\mathrm{N}=4887)$

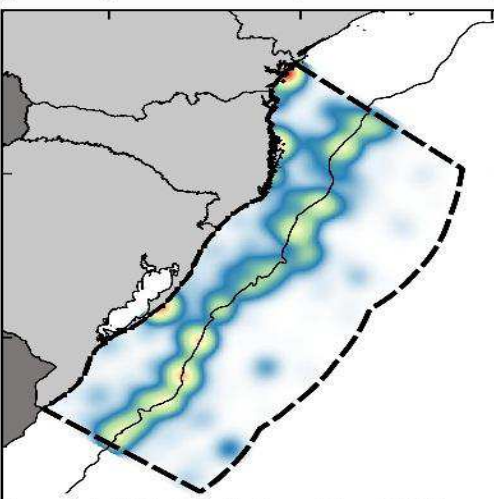

(F) GBIF: Pelágicos $(\mathrm{N}=6570)$
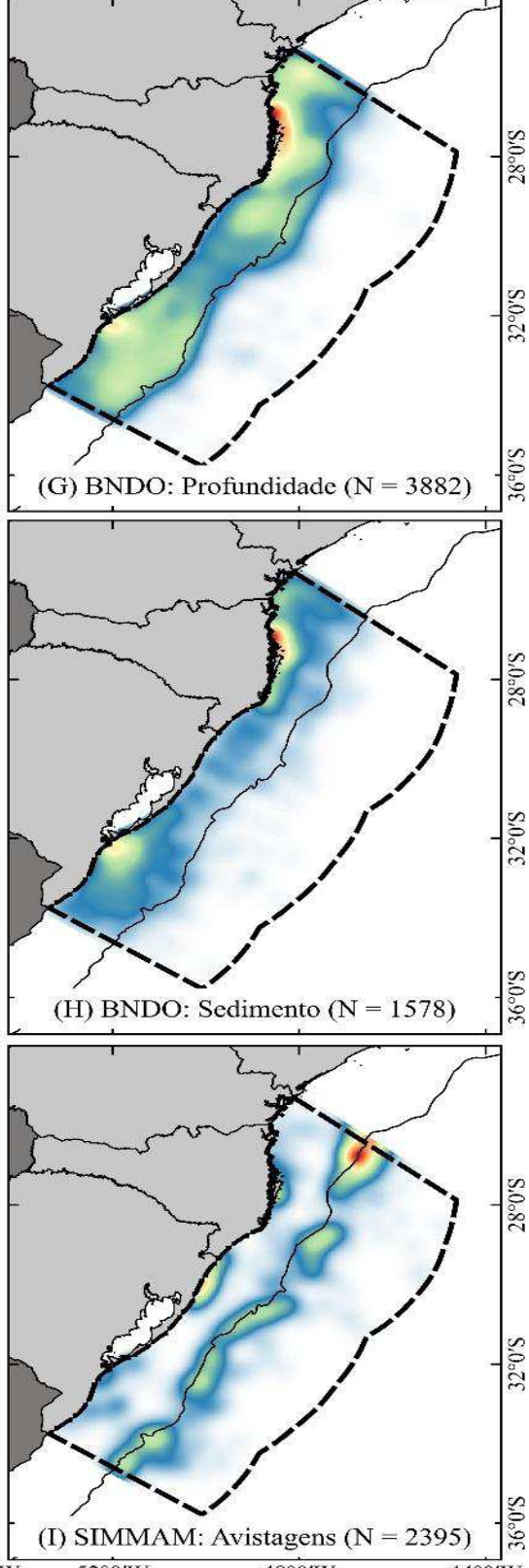

FIGURA 3 - Densidade kernel normalizada (mapa de cores) de dados de alguns repositórios na Zona Econômica-Exclusiva do Sul do Brasil (linha pontilhada) e quebra teórica da plataforma continental (isóbata de $200 \mathrm{~m}$, linha contínua).

FONTE: Elaboração própria. 


\subsubsection{Zonas de Restrição, Manejo e Regulação}

O tema Zonas de Restrição, Manejo e Regulação foi definido no INSPIRE de forma genérica como áreas gerenciadas, reguladas ou utilizadas de forma definida por legislação nos diversos níveis de governo (local, regional, nacional, continental).

Para o caso brasileiro, este tema incluiria, por exemplo, áreas de dragagem e descarte de sedimentos, áreas de exclusão de pesca, zonas de amortecimento de unidades de conservação, áreas restritas para navegação, entre outras áreas com usos restringidos pela legislação vigente. Não existe no Brasil uma base de dados com o mapeamento destas áreas. É possível mapear estas zonas por meio de coordenadas disponíveis na legislação e com a aplicação de procedimentos simples de geoprocessamento (buffers, intersecções, etc.).

O Centro Nacional de Pesquisa e Conservação da Biodiversidade Marinha do Sudeste e Sul (CEPSUL) mapeou as zonas de exclusão de pesca do sudeste e do sul do Brasil ${ }^{19}$, porém, este dado é disponibilizado em formato de figura, não sendo possível a sua utilização direta como descritor espacial para o PEM. O PREPS disponibiliza estas informações em formato vetorial, porém, o acesso a estes dados é restrito, não tendo sido possível a sua obtenção para avaliação neste trabalho.

Outras zonas com manejo específico podem ser obtidas nos planos de desenvolvimento portuário (ex. bacias de evolução, áreas de dragagem e descarte) e áreas de navegação restritas definidas em cartas náuticas. $\mathrm{O}$ mapeamento de polígonos definidos em licenças ambientais e Estudos de Impacto Ambiental aprovados também seriam desejáveis para esta camada de Zonas de Restrição, Manejo e Regulação. Entretanto, sem uma política de governo que exija o cadastramento destas zonas pelos órgãos licenciadores (federais, estaduais e municipais) em um repositório de dados geoespaciais, parecem ser inviáveis o processo de pesquisa das licenças ambientais nos sistemas dos diferentes órgãos, a transformação em informação espacial e a utilização no PEM.

\subsubsection{Regiões Marinhas}

As Regiões Marinhas são definidas como áreas com características físicas semelhantes, como tipo de sedimento, substrato, profundidade e temperatura (European Commission Joint Research Center, 2013). Dados de tipo de substrato (sedimento) estão disponíveis no Banco Nacional de Dados Oceanográficos (BNDO), na forma de percentuais de areia, silte e cascalho. O BNDO é gerido pela Marinha do Brasil e o acesso aos dados se dá por meio de solicitação e justificativa para o uso dos dados. Estes são disponibilizados na forma de tabelas, com coordenadas geográficas em um formato que junta graus e minutos decimais sem separadores, tornando difícil a inserção destes dados nos softwares convencionais de Sistemas de Informações Geográficas (SIG). Portanto, é necessário desenvolver rotinas para a importação das tabelas e a transformação em dados espaciais, o que limita a utilização destes dados por usuários iniciantes em SIG.

\footnotetext{
${ }^{19} \mathrm{http}: / /$ www.icmbio.gov.br/cepsul/areas-protegidas/area-de-exclusao-a-pesca.html
} 
Na ZEE do sul do Brasil, os dados de tipo sedimento estão distribuídos principalmente na plataforma continental, com grandes lacunas entre o litoral norte do Rio Grande do Sul e o Cabo de Santa Marta (SC). Áreas de alta densidade de pontos se localizam no trecho adjacente ao estuário da Lagoa dos Patos e entre Florianópolis (SC) e São Francisco do Sul (SC) (Figura 3 - H).

Dados de temperatura superficial do mar (TSM) são de fácil aquisição por meio de sensores remotos, que permitem uma visão sinótica de alta resolução temporal (mais de uma imagem por dia). O problema de cobertura de nuvens, que gera lacunas em dados de TSM diários, é minimizado quando se utiliza agregação por intervalos de tempo (ex. médias mensais ou sazonais). Entretanto, é necessário definir como analisar a série de dados para que possam ser utilizados como descritores para o PEM. Devido à diversidade de repositórios de dados de sensoriamento remoto dos oceanos para parâmetros como clorofila e TSM, estes não foram analisados neste trabalho. Além disso, deve-se destacar que esses dados se referem apenas à camada superficial dos oceanos, sendo necessária a obtenção de dados oceanográficos in situ em outros repositórios para a definição desta propriedade na coluna d'água.

\subsubsection{Habitats e Biótopos}

$\mathrm{O}$ S biótopos representam áreas geográficas com comunidades biológicas distintas associadas a fatores ambientais como tipo de substrato, profundidade ou temperatura (Davies et al., 2017). Na INSPIRE, os habitats e biótopos são classificados por meio da chave de classificação da European Nature Information System (EUNIS), que leva em consideração fatores como penetração da luz, substrato e profundidade para a identificação de habitats, refinando a classificação com a incorporação de dados de comunidades biológicas para o nível de biótopos (Commission of the European Communities, 1991). No Brasil, a maior parte das iniciativas de classificação de habitats é em escala local (portos, unidades de conservação) e de âmbito ainda acadêmico. Mussi et al. (2017) sugerem, para a plataforma continental de Santa Catarina, uma abordagem metodológica de classificação até o nível de habitats na camada de fundo a partir de dados de substrato, penetração de luz (estimada a partir de imagens do sensor MODIS) e energia de ondas, obtida por modelagem hidrodinâmica. Esta metodologia é passível de adaptação e replicação para a plataforma continental do sul do Brasil, desde que estejam disponíveis os dados de entrada.

Para o refinamento dos habitats e a delimitação dos biótopos é necessária a incorporação de dados de ocorrência de organismos. Foram consultados dois repositórios de dados internacionais de biodiversidade, o Ocean Biogeographical Information System (OBIS) e o Global Biodiversity Information Facility (GBIF).

O OBIS é um repositório global de registros de ocorrência de animais marinhos e disponibiliza um grande número de registros na ZEE do sul do Brasil $(n=35560)$. A maior parte dos dados disponíveis (44\%) no OBIS foi coletada no contexto do Programa de Avaliação do Potencial Sustentável de Recursos Vivos na Zona Econômica Exclusiva (REVIZEE). A maioria dos registros foi obtida em três "pulsos": 1973, 1995 e 2006, sendo que nos últimos 7 anos o número de registros inseridos no OBIS para a ZEE do sul do Brasil é muito pequeno. 
O GBIF é uma infraestrutura de pesquisa de dados abertos com o objetivo de prover acesso a dados sobre todos os tipos de vida na Terra. O Brasil é um país associado ao GBIF desde 2012 e criou o Sistema de Informação sobre a Biodiversidade Brasileira (SIBBr) como um nó do GBIF. Apesar de possuir um foco nas áreas terrestres, foram encontradas no GBIF ocorrências de espécies marinhas na ZEE do sul do Brasil ( $n=13972)$.

Além das lacunas espaciais e temporais, também é possível perceber nos dados disponíveis no OBIS e no GBIF uma grande diferença no número de registros em relação às classes e modo de vida da fauna, com maior ocorrência de organismos pelágicos, em relação aos organismos bentônicos e planctônicos (Figura 3).

Os registros de organismos pelágicos do OBIS se encontram bem distribuídos na plataforma continental do sul do Brasil, com uma lacuna na região costeira do norte de Santa Catarina e em todo o litoral do Paraná, e alta densidade de registros na plataforma continental ao sul da desembocadura da Lagoa dos Patos (Figura 3 - C). Já no GBIF, a maior densidade de registros pelágicos está na quebra da plataforma continental (Figura 3 - F), com alguns hotspots costeiros no trecho da Lagoa do Peixe (RS), norte da Ilha de Santa Catarina (SC) e Paranaguá (PR).

Para a fauna bentônica do OBIS, existem pequenas áreas de alta densidade nas proximidades do estuário da Lagoa dos Patos (RS) e da Baía de Paranaguá (PR) e alguns registros ao longo da quebra da plataforma continental, mas grandes lacunas entre as isóbatas de 30 a 150 m (Figura 3 - A). No GBIF, foram encontradas altas densidades ao longo da quebra da plataforma e Paranaguá (Figura 3 - D).
Os dados de organismos planctônicos são mal distribuídos nos dois repositórios, com alta densidade no OBIS em uma faixa no norte de Santa Catarina (Figura 3 - B) e no GBIF nas proximidades de Paranaguá (Figura 3 - E).

Ainda com relação à disponibilização de registros de organismos marinhos, é importante valorizar os esforços na sistematização e na distribuição de dados de ocorrência de mamíferos marinhos pelo Sistema de Apoio ao Monitoramento de Mamíferos Marinhos (SIMMAM). O SIMMAM, desenvolvido pela Universidade do Vale do Itajaí e cedido ao ICMBio, foi criado com o objetivo de coletar e armazenar informações georreferenciadas sobre avistagens, capturas incidentais e encalhes de mamíferos aquáticos e possui dados provenientes de dezenas de instituições que trabalham com mamíferos aquáticos. Os dados de avistagens de mamíferos se distribuem principalmente ao longo da quebra da plataforma continental e talude, assim como na área costeira no norte do Rio Grande do Sul e nas proximidades da Área de Proteção Ambiental da Baleia Franca (Figura 3 - I).

A falta de homogeneidade na distribuição de dados de fauna e flora em zonas marinhas é um fator restritivo para a utilização destes dados como descritores para o PEM, uma vez que os hotspots encontrados podem ter mais influência dos esforços de amostragem de projetos de pesquisa específicos do que da importância para conservação.

\subsubsection{Infraestrutura Industrial e de Produção}

No INSPIRE, o tema Infraestrutura Industrial e de Produção é bastante abrangente e agrega dados relacionados à produção e à indústria, assim como 
informações de resumo sobre os impactos ambientais relacionados a estas atividades. Este tema é utilizado para a formação de diversos descritores de usos e atividades humanas, como a pesca industrial, produção de energia elétrica, extração de petróleo e gás, entre outras.

No Brasil, o Programa Nacional de Rastreamento de Embarcações Pesqueiras por Satélite (PREPS), instituído por lei (Brasil, 2006), obriga embarcações da frota industrial com arqueação bruta maior do que 15 metros a instalarem transmissores de satélite para a localização das embarcações em tempo real. O acesso aos dados é restrito a servidores da Marinha do Brasil, do Ministério do Meio Ambiente, do Instituto Brasileiro do Meio Ambiente e dos Recursos Naturais Renováveis (IBAMA), do Instituto Chico Mendes de Conservação da Biodiversidade (ICMBio) e do Ministério da Agricultura, Pecuária e Abastecimento (MAPA).

O PREPS armazena dados essenciais para o diagnóstico de uso da ZEE pela pesca industrial, mas não há um processo instituído de solicitação de acesso aos dados por pesquisadores. A restrição do acesso aos dados envolve questões de "segredo industrial", considerando que os locais de pesca são vantagens industriais que os armadores e pescadores possuem em relação aos seus concorrentes. Porém, o grau de detalhe ou granulação dos dados coletados pode afetar a disposição dos atores sociais para compartilhar a informação (Shucksmith \& Kelly, 2014). Os órgãos responsáveis pelo PREPS podem optar por disponibilizar os dados agregados na forma de, por exemplo, mapas de densidade sazonais por arte de pesca, sem a identificação dos barcos de pesca e, portanto, sem prejuízo aos pescadores.

Alguns autores (St. Martin \& Hall-Arber, 2008; Littaye et al., 2016; Strickland-Munro et al.,
2016) ressaltam que, enquanto dados das condições físicas e biológicas são abundantes, dados dos usos na zona marinha são escassos e muitas vezes pouco confiáveis. Portanto, o PREPS pode vir a ser um diferencial positivo do Brasil no processo de PEM. Uma política de acesso aos dados do PREPS é de grande importância para o avanço do PEM no Brasil, já que auxiliaria no diagnóstico de conflitos de usos.

O processamento dos dados do PREPS não é trivial, sendo necessários vários filtros para eliminar pontos espúrios, bem como para separar registros de navegação e desembarques dos registros de atividade da pesca. As regiões de baixa densidade de pontos do PREPS não devem ser consideradas como lacunas de dados, e sim regiões efetivamente menos utilizadas pela pesca industrial e, por isso, não foram analisadas neste artigo.

\subsubsection{Redes de Transporte}

O tema Redes de Transporte abarca as redes de transporte terrestre, aquático e aéreo e as infraestruturas relacionadas (portos, aeroportos, estações de trens e ônibus, etc.) e é utilizado para elaboração dos descritores relacionados à navegação comercial. O Plano Nacional de Logística e Transporte contém dados geoespaciais de rotas de navegação, ferrovias e rodovias, porém, a única CI encontrada na INDE sobre este tema é a de localização dos portos. As CIs de transporte terrestres são relevantes para o PEM, porém fogem do escopo deste trabalho, que se concentrou na busca de informações no meio marinho.

Para os descritores do uso para navegação, é possível utilizar dados disponíveis no Marine Traffic. Esta plataforma coleta e disponibiliza 
dados provenientes do Sistema Automático de Identificação (AIS, em inglês), que é um sistema de rastreamento que utiliza comunicação entre navios para obtenção da localização dos mesmos por todo o mundo. Da mesma forma que o PREPS, os dados de AIS representam pontos de rastreamento contínuo e as áreas de baixa densidade não devem ser consideradas como lacunas espaciais de dados, e sim regiões efetivamente pouco utilizadas para navegação.

Foi realizado junto àquela empresa um orçamento para a aquisição de posições de navios na ZEE do sul do Brasil nos últimos de cinco anos, que teria um custo de cerca de US\$ 9.000, valores elevados para uma pesquisa acadêmica, mas módicos para uma iniciativa governamental de PEM. Por outro lado, a empresa disponibiliza mapas de densidade anuais gratuitos, sendo possíveis o georreferenciamento e a transformação destes em dados qualitativos, identificando as principais rotas de navegação, bem como zonas de atracação ou espera de navios.

\section{Considerações finais}

A detecção de lacunas é apontada por alguns autores como um dos primeiros passos para o PEM (Ardron et al., 2008; Flannery \& Cinnéide, 2012; Shucksmith \& Kelly, 2014; Smith, 2015). A identificação das lacunas espaciais nos dados permite a elaboração de mapas de incertezas, que podem ser utilizados para o direcionamento de esforços de coleta, disponibilização ou harmonização de dados para preencher estas lacunas.

No Brasil, deve ser dada prioridade para a construção do tema "Zonas de Restrição, Manejo e Regulação", que possui grande relevância devido ao número de descritores para o PEM relacionados. Este tema pode ser elaborado por meio da espacialização de normas e legislação relacionadas aos usos na zona marinha.

Não há ainda a consolidação do conhecimento científico nos temas Habitats e Biótopos e Regiões Marinhas, que são utilizados para um grande número de descritores para o PEM. Por outro lado, dados existentes no Brasil, porém dispersos, podem ser utilizados para a construção destes temas. Isto evidencia a necessidade de investimento em esforços de integração, disponibilização e harmonização dos dados, aumentando a aplicabilidade para o PEM dos dados produzidos pela comunidade científica.

O BNDO apresenta problemas estruturais para que alcance o status de uma IDE: falta de padronização e metadados, impossibilidade de pesquisa e visualização de dados e solicitação dos mesmos por e-mail, o que desencoraja a utilização deste como repositório oficial para o PEM. É crucial uma reformulação estrutural do BNDO ou a incorporação dos dados ali depositados a uma infraestrutura alternativa/complementar. A rica base ali disponível deve ser estruturada de modo a seguir os padrões internacionais e estabelecidos pela INDE para que se tenha uma efetiva infraestrutura de dados para as áreas marinhas no Brasil, possibilitando o resgate de dados em três dimensões espaciais que considere, ainda, a alta variabilidade temporal inerente aos sistemas costeiros e oceânicos (Bartlett, 2000).

O Brasil possui, ainda, inúmeras instituições e pesquisadores que coletam, analisam e publicam dados marinhos derivados de projetos específicos na forma de artigos científicos e relatórios. Entretanto, há uma carência de estruturas que facilitem e incentivem a disponibilização e padronização destes dados. 
Pode-se ressaltar que o PEM deve ser um processo adaptativo, capaz de incorporar novas fontes de dados e métodos de análise, o que demanda a inclusão contínua de novos provedores. Com o avanço do PEM e o estabelecimento de demandas e incentivos governamentais, espera-se que mais instituições passem a compartilhar dados marinhos e alimentem repositórios nacionais e internacionais, garantindo o uso da melhor informação científica disponível para o PEM.

\section{Agradecimentos}

Ao Instituto Federal de Educação, Ciência e Tecnologia do Rio Grande do Sul (IFRS) pelo fomento através do afastamento para pós-graduação do primeiro autor.

\section{Referências}

Ardron, J.; Gjerde, K.; Pullen, S.; Tilot, V. Marine spatial planning in the high seas. Marine Policy, 32(5), 832-839, 2008. doi:10.1016/j.marpol.2008.03.018

Bartlett, D. J. Working on the frontiers of science: Applying GIS to the coastal zone. In: Wright, D.; Bartlett, D. (Eds.). Marine and Coastal Geographic Information Systems. London: Taylor \& Francis, 2000.

Brasil. Instrução Normativa Interministerial $n^{\circ} 2$, de 4 de setembro de 2006. Institui o Programa Nacional de Rastreamento de Embarcações Pesqueiras por Satélite - PREPS para fins de monitoramento, gestão pesqueira e controle das operações da frota pesqueira permissionada pela Secretaria Especial de Aquicultura e Pesca da Presidência da República - SEAP/PR. Brasília: DOU de 15/09/2006.

Collie, J. S.; (Vic) Adamowicz, W. L.; Beck, M. W.; Craig, B.; Essington, T. E.; Fluharty, D.; Rice, J.; Sanchirico, J. M. Marine spatial planning in practice. Estuarine and
Coastal Shelf Science, 117, 1-11, 2013. doi: 10.1016/j. ecss.2012.11.010

Commission of the European Communities. Habitats of the European Community. CORINE biotopes manual. Luxemburgo: Commission of the European Communities, 1991.

Conselho Nacional de Cartografia. INDE: Infraestrutura Nacional de Dados Espaciais, s/d. Disponível em: $<$ http:// www.inde.gov.br/>. Acesso em: abr. 2017.

Davies, J. S.; Guillaumont, B.; Tempera, F.; Vertino, A.; Beuck, L.; Ólafsdóttir, S. H.; Smith, C. J.; Fossa, J. H.; van den Beld, I. M. J.; Savini, A.; Rengstorf, A.; Bayle, C.; Bourillet, J. F.; Arnaud-Haond, S.; Grehan, A. A new classification scheme of European cold-water coral habitats: implications for ecosystem-based management of the deep sea. Deep Sea Research Part II, in press, 2017. doi: 10.1016/j.dsr2.2017.04.014

De Freitas, D. M.; Xavier, L. Y.; Shinoda, D. Relatório do Seminário Internacional: Planejamento Integrado do Espaço Marinho. Brasília: Ministério do Meio Ambiente, 2014. doi: 10.13140/2.1.3699.4088

Donlon, C. J.; Martin, M.; Stark, J.; Roberts-Jones, J.; Fiedler, E.; Wimmer, W. The Operational Sea Surface Temperature and Sea Ice Analysis (OSTIA) system. Remote Sensing of Environment, 116, 140-158, 2012. doi: 10.1016/j. rse.2010.10.017

Douvere, F.; Maes, F.; Vanhulle, A.; Schrijvers, J. The role of marine spatial planning in sea use management: The Belgian case. Marine Policy, 31, 182-191, 2007. doi: 10.1016/j.marpol.2006.07.003

Ehler, C.; Douvere, F. Marine spatial planning: A step-by-step approach toward Ecosystem-based Management. IOC Manual. Paris: Programme, Intergovernmental Oceanographic Commission and Man and the Biosphere - UNESCO, 2009.

European Commission. MSP Data Study Executive Summary. Evaluation of data and knowledge gaps to implement MSP. Bruxelas: European Commission, 2016. doi: $10.2826 / 25289$

European Commission. INSPIRE: Infraestructure for spatial data in Europe, s/d. Disponível em: $<$ http://inspire.ec.euro- 
pa.eu>. Acesso em: maio 2017.

European Commission Joint Research Centre. D2.8.III.16 Data Specification on Sea Regions - Technical Guidelines. Bruxelas: European Commission, 2013.

Flannery, W.; Cinnéide, M. O. A roadmap for marine spatial planning: A critical examination of the European Commission's guiding principles based on their application in the Clyde MSP Pilot Project. Marine Policy, 36, 2012. doi: 10.1016/j.marpol.2011.06.003

Gandra, T. B. R.; Bonetti, J.; Scherer, M. E. G. Utilização do software de apoio à decisão MARXAN para a priorização de áreas de conservação na Zona Econômica-Exclusiva no Sul do Brasil. In: Anais do XVIII Simpósio Brasileiro de Sensoriamento Remoto - SBSR. Santos: INPE, 2017, 6186-6193. Disponível em: <https://proceedings.galoa.com.br/sbsr>.

Gern, F. R.; Longarete, C.; Christofidis, M.; Rosa, F. D.; Maçaneiro, L. R.; Polette, M. Diagnóstico da zona de uso aquaviário e portuário de Itajaí - ZUAP (SC): bases para planejamento espacial marinho (PEM) local. Desenvolvimento e Meio Ambiente, 40, 459-482, 2017. doi: 10.5380/ dma.v40i0.47850

Halpern, B. S.; Diamond, J.; Gaines, S.; Gelcich, S.; Gleason, M.; Jennings, S.; Lester, S.; Mace, A.; McCook, L.; McLeod, K.; Napoli, N.; Rawson, K.; Rice, J.; Rosenberg, A.; Ruckelshaus, M.; Saier, B.; Sandifer, P.; Scholz, A.; Zivian, A. Near-term priorities for the science, policy and practice of Coastal and Marine Spatial Planning (CMSP). Marine Policy, 36(1), 198-205, 2012. doi: 10.1016/j.marpol.2011.05.004

Littaye, A.; Lardon, S.; Alloncle, N. Stakeholders' collective drawing reveals significant differences in the vision of marine spatial planning of the western tropical Pacific. Ocean \& Coastal Management, 130, 2016 doi: 10.1016/j. ocecoaman.2016.06.017

Masser, I. GIS Worlds: Creating Spatial Data Infrastructures. Redlands: ESRI Press, 2005.
MMA - Ministério do Meio Ambiente. Áreas Prioritárias para Conservação, Uso Sustentável e Repartição de Benefícios da Biodiversidade Brasileira: Atualização, 2007. Disponível em: <http://areasprioritarias.mma.gov.br/>. Acesso em: ago. 2017.

Mussi, C. S.; Sperb, R. M.; Bonetti, J. Mapeamento da paisagem marinha e costeira de Santa Catarina através de chaves classificatórias de habitats. In: Anais do X Encontro Nacional de Gerenciamento Costeiro - X ENCOGERCO. Rio Grande: FURG, 2017, 43-44. Disponível em: <www. encogerco.com.br>.

Shucksmith, R.; Gray, L.; Kelly, C.; Tweddle, J. F. Regional marine spatial planning - The data collection and mapping process. Marine Policy, 50, 1-9, 2014. doi: 10.1016/j.marpol.2014.05.012

Shucksmith, R. J.; Kelly, C. Data collection and mapping: Principles, processes and application in marine spatial planning. Marine Policy, 50, 27-33, 2014. doi: 10.1016/j. marpol.2014.05.006

Smith, G. Creating the spaces, filling them up. Marine spatial planning in the Pentland Firth and Orkney Waters. Ocean \& Coastal Management, 116, 132-142, 2015. doi: 10.1016/j.ocecoaman.2015.07.003

St. Martin, K.; Hall-Arber, M. The missing layer: Geo-technologies, communities, and implications for marine spatial planning. Marine Policy, 32, 779-786, 2008. doi: 10.1016/j. marpol.2008.03.015

Stamoulis, K. A.; Delevaux, J. M. S. Data requirements and tools to operationalize marine spatial planning in the United States. Ocean \& Coastal Management, 116, 214-223, 2015. doi: 10.1016/j.ocecoaman.2015.07.011

Strickland-Munro, J.; Kobryn, H.; Brown, G.; Moore, S. A. Marine spatial planning for the future: Using Public Participation GIS (PPGIS) to inform the human dimension for large marine parks. Marine Policy, 73, 15-26, 2016. doi: 10.1016/j.marpol.2016.07.011 